\title{
Transplantation of Autologous Bone Marrow Mononuclear Cells Regulates Inflammation in a Rabbit Model of Carotid Artery Atherosclerosis
}

\author{
Kefei Cui ${ }^{a}$ Min Wang ${ }^{a}$ Lie Yu ${ }^{b}$ Xiao Ren ${ }^{c}$ Hui Cui ${ }^{a}$ Xiao Fang Yu \\ Suyun $\mathrm{Hou}^{\mathrm{a}} \mathrm{Chao} \mathrm{Fu}^{\mathrm{a}}$ Jian Wang ${ }^{\mathrm{d}}$ \\ ${ }^{a}$ Department of Ultrasound, The First Affiliated Hospital of Zhengzhou University, and ${ }^{b}$ Department of \\ Neurology, The Fifth Affiliated Hospital of Zhengzhou University, Zhengzhou, 'Department of Biochemistry and \\ Molecular Biology, The First Clinical Medical College of Nanchang University, Nanchang, China; ${ }^{d}$ Department of \\ Anesthesiology/Critical Care Medicine, School of Medicine, Johns Hopkins University, Baltimore, Md., USA
}

\section{Key Words}

Atherosclerosis · Autologous bone marrow mononuclear cells $\cdot$ Inflammatory responses

\begin{abstract}
Objective: It is well known that inflammation plays key roles in the development of atherosclerosis and that the transplantation of bone marrow mononuclear cells (BMMNCs) can suppress inflammation in rodent models of ischemic diseases. Here, we explored whether transplantation of autologous BMMNCs could prevent the progression of atherosclerosis by the alleviation of inflammatory responses in a rabbit model of carotid artery atherosclerosis. Methods and Results: The atherosclerotic rabbit model was established by air desiccation followed by a high-cholesterol diet for 8 weeks. Then, $1 \times 10^{7}$ BMMNCs labeled with BrdU or an equal volume of vehicle were injected into the rabbits via the ear vein. Using an ultrasonographic imaging method, we found that autologous BMMNC treatment significantly decreased the area of atherosclerotic plaques compared to the vehicletreated group $(p<0.05)$. The results were further confirmed by hematoxylin-eosin staining. RT-PCR results demonstrated that BMMNC treatment significantly reduced the expression of interleukin (IL)- 6 and CD147 but increased the expression
\end{abstract}

of IL-10 and transforming growth factor- $\beta$ compared with vehicle treatment $(p<0.05)$, which was consistent with Western blot results. Conclusions: Transplantation of autologous BMMNCs delays the development of atherosclerosis, most probably via the attenuation of inflammatory responses, which could be a new approach for treating carotid atherosclerosis.

(c) 2016 S. Karger AG, Basel

\section{Introduction}

Atherosclerosis (AS) leads to cardiovascular disease which gives rise to fatal clinical outcomes, such as stroke or myocardial infarction [1]. The atherosclerotic lesion derives from the declination of the number of endothelial progenitor cells (EPCs) or its function $[2,3]$ followed by lipid accumulation in macrophages and vascular smooth muscle cells (VSMCs) [4].

Inflammation is the central event at all stages of AS. Circulating immune cells are involved in the atherosclerotic process through the secretion of a large number of cytokines [5]. They can be classified into proinflammatory and anti-inflammatory cytokines $[5,6]$. The former is characterized with IL-6 and CD147 [5-7]. IL-6 can pro- 
mote $\mathrm{T}$ lymphocyte differentiation and the production of inflammatory mediators including TNF- $\alpha$ and IL- $1 \beta[5$, 6]. CD147 is an inducer of extracellular matrix metalloproteinases (MMPs), including MMP-9 [7]. The upregulation of MMP-9 is a direct cause of the development and rupture of atherosclerotic plaques [5]. The latter primarily consists of IL-10 and transforming growth factor (TGF)- $\beta[5,8-10]$. IL-10 is a prototypic anti-inflammatory cytokine, which can inhibit the activation of macrophages, activity of MMP-9, release of proinflammatory cytokines and expression of cyclooxygenase-2 in lipidloaded and activated macrophage foam cells $[8,10]$. TGF- $\beta$ can reduce $T$ cell infiltration and increase collagen production in the plaques $[9,10]$. Therefore, maintenance of the balance of proinflammatory and anti-inflammatory mediators is recognized as an ideal approach for suppressing the development of AS.

The therapeutic use of bone marrow mononuclear cells (BMMNCs) for ischemic diseases is currently under clinical investigation [11-13]. Given secretion of IL-10 and TGF- $\beta$, BMMNCs have been shown to exhibit protective effects on myocardial infarction and cerebral infarction by regulation of proinflammatory and anti-inflammatory cytokines $[14,15]$. However, there is no consensus on the role of BMMNCs in the development of AS [16-18]. One study showed that intracoronary injection of unfractionated BMMNCs in patients with acute STelevation myocardial infarction does not accelerate AS progression [16]. In contrast, 2 other studies reported that BMMNCs promote atherosclerotic plaque development in diabetic apoE $\mathrm{E}^{-/-}$mice [17] and wild-type mice [18]. On analysis of those studies $[16,18]$ we found that the BMMNCs used were collected from syngeneic mice instead of autologous animals. In this study, we established an atherosclerotic plaque model in the carotid artery of rabbits by air desiccation. We aimed to investigate the effects of autologous BMMNCs on the area of atherosclerotic plaques and explore the underlying mechanisms.

\section{Materials and Methods}

\section{Carotid Injury and Treatment}

New Zealand white rabbits (male, $1.5-1.7 \mathrm{~kg}, \mathrm{n}=78$ ) were purchased from the Animal Experimental Center of Zhengzhou University. All protocols were approved by the Animal Care and Use Committee of Zhengzhou University and were in accordance with the institutional guidelines. These animals were divided into 4 groups. In the naive group $(n=6)$ rabbits received a conventional diet for 10 weeks and were not subjected to any operations. In the sham operated group $(n=24)$ the middle of the neck of these rabbits was incised and then sutured; the animals were fed a high-cholesterol diet for 10 weeks. In the vehicle treatment group $(n=24)$ rabbits were subjected to air desiccation and were given a high-cholesterol diet for 8 weeks; they were then treated with $1 \mathrm{ml}$ of phosphate-buffered saline (PBS) at the end of the eighth week and were fed a high-cholesterol diet for the next 2 weeks. Finally, the BMMNC treatment group ( $\mathrm{n}=$ 24) consisted of rabbits that were subjected to air desiccation and fed a high-cholesterol diet for 8 weeks; they were then intravenously treated with $1 \times 10^{7}$ BMMNCs at the end of the eighth week and were given a high-cholesterol diet for next 2 weeks. These rabbits were sacrificed on 1, 3, 7, and 14 days after BMMNCs treatment.

The atherosclerotic plaque model was established as previously described [19]. In brief, rabbits were anesthetized by intramuscular injection of $0.2 \mathrm{ml} / \mathrm{kg}$ of xylazine hydrochloride. The left common carotid arteries of the rabbits were surgically exposed and temporarily occluded using two microvascular clamps with a $3-\mathrm{cm}$ interval. The endothelia of the arterial lumen were dried by nitrogen air flow for $8 \mathrm{~min}$ at $80 \mathrm{ml} / \mathrm{min}$ via needle puncture. After desiccation, the clamps were removed to restore blood flow and the cuts were sutured. The rabbits were fed the diets previously described.

\section{Bone Marrow Cell Processing}

Experimental animals underwent bone marrow puncture 8 weeks after carotid artery injury. The rabbits were fastened to the work table after being anaesthetized through xylazine hydrochloride injection. The puncture point was located in the tibial medial, $1 \mathrm{~cm}$ under the anterior tibialis tuberosity. The bone marrow aspirates were washed in PBS and centrifuged (3,000 rpm, $10 \mathrm{~min}$ ). They were then added to an equal amount of Ficoll-Paque Plus (Tianjin TBD Ltd., Beijing, China) and centrifuged at 2,500 rpm for $30 \mathrm{~min}$. The white film was extracted and washed 3 times in PBS. A total of $5 \mathrm{ml}$ of DMEM containing $10 \mu \mathrm{l}$ of penicillin-streptomycin solution, $100 \mu \mathrm{l}$ of bovine serum albumin and $50 \mu \mathrm{l}$ of bromodeoxyuridine (BrdU) solution (final concentration $12 \mathrm{~g} / \mathrm{ml}$; Sigma-Aldrich, St. Louis, Mo., USA) was added to the cell precipitation and medium. After incubating overnight at $37^{\circ} \mathrm{C}$, the BMMNCs were collected and filtered through a 500 mesh strainer (diameter $=25 \mu \mathrm{m})$, washed with PBS and counted using a hemocytometer. Finally, $1 \times 10^{7}$ cells labeled with BrdU were intravenously injected via the ear vein. Vehicle-treated animals were injected with an equal volume of PBS.

\section{Vessel Harvesting}

The carotid arteries were fixed in $4 \%$ paraformaldehyde for $12 \mathrm{~h}$ at $4^{\circ} \mathrm{C}$, then embedded in Tissue-Tek OCT compound (Sakura Finetechnical, Tokyo, Japan), snap-frozen, and stored at $-80^{\circ} \mathrm{C}$. Samples were sectioned at a thickness of $5 \mu \mathrm{m}$ and placed on poly-L-lysine-coated slides for immunofluorescent and hematoxylin-eosin (HE) staining.

\section{Assessment of AS}

The extent of AS was quantified by calculating the lesion size in the carotid artery [20].

\section{Ultrasonography}

The carotid arteries of rabbits fed a high-cholesterol or conventional diet for 8 or 10 weeks were examined using a Siemens Se- 
quoia 512 ultrasound scanner with a 6- to $14-\mathrm{MHz}$ linear probe (Siemens, Mountain View, Calif., USA). The average plaque diameter was measured.

\section{Immunofluorescence Double Staining}

Vessel specimens from rabbits on days 1 and 14 after transplantation were cut into $5-\mu \mathrm{m}$ sections and then treated with $2 \mathrm{M} \mathrm{HCl}$ for $30 \mathrm{~min}$ and washed before incubating with blocking buffer. Endogenous peroxidase was blocked with 3\% hydrogen peroxide for $5 \mathrm{~min}$, and nonspecific binding was blocked in blocking buffer (5\% normal goat serum, $0.3 \%$ Triton $\mathrm{X}$ in PBS) for $1 \mathrm{~h}$ at room temperature. Sheep anti-BrdU polyclonal antibody (1:100; Abcam, Cambridge, Mass., USA) and mouse anti-rabbit monoclonal antibody against CD31 (1:100; Abcam) were used as primary antibodies. Sections were incubated in the primary antibodies overnight at $4^{\circ} \mathrm{C}$ and then with Alexa Fluor 488-conjugated donkey anti-sheep IgG $\mathrm{H}+\mathrm{L}$ antibody (1:800; Abcam) and CFL594-conjugated donkey anti-mouse IgG (1:200; Santa Cruz Biotechnology, Santa Cruz, Calif., USA) for $2 \mathrm{~h}$ at room temperature for visualization of antibody binding. Photomicrographs were obtained with an epifluorescence deconvolution microscope (Olympus CKX41, Olympus, Japan). Cells positive for BrdU were counted as BMMNC-derived cells.

\section{$R T-P C R$}

RNA was isolated (Beijing ComWin Biotech, Beijing, China) and analyzed by RT-PCR. Each PCR assay was performed in duplicate using cDNA samples isolated from individual rabbits $(\mathrm{n}=$ 6 per group). The primers used for amplification were as follows. For IL-6, the forward primer was $5^{\prime}$-TGAGACCTGCCTGCTGAGA- $3^{\prime}$, and the reverse primer was $5^{\prime}$-TGAGGGTGGCTTCTTCATTC-3' (product band, $185 \mathrm{bp}$ ). For IL-10, the forward primer was $5^{\prime}$-GAGAACCACAGTCCAGCCAT-3', and the reverse primer was $5^{\prime}$ - CATGGCTTTGTAGACGCCTT-3' (product band, $179 \mathrm{bp}$ ). For CD147, the forward primer was $5^{\prime}$-CAGAATGACAAAGGCAAGAACG-3', and the reverse primer was 5'-GGCTTGGGAGTGACTTGAGG-3' (product band, 269 bp). For TGF- $\beta$, the forward primer was $5^{\prime}$-CCGCAACAACACCCACA- $3^{\prime}$, and the reverse primer was $5^{\prime}$-CGGCACCTCATCATCACTT- $3^{\prime}$ (product band, 112 bp). For GAPDH, the forward primer was $5^{\prime}$-GAACGGGAAACTCACTGGCAT- $3^{\prime}$, and the reverse primer was $5^{\prime}$-CCTTCTTGATGTCGTCATACTTAGC-3' (product band, $110 \mathrm{bp}$ ). Twenty-microliter mixtures were electrophoresed in $1.5 \%$ agarose gel. Values were normalized to the GAPDH band by Quantity One software.

\section{Western Blot}

Vessels homogenized in radioimmunoprecipitation assay (RIPA) lysis buffer (Boster, Wuhan, China) were clarified by centrifugation $\left(14,000 \mathrm{rpm}, 5 \mathrm{~min}, 4^{\circ} \mathrm{C}\right)$. A standard Western blotting protocol was followed. The samples were separated by $4 \%$ stacking gel and $10 \%$ separating gel was transferred onto polyvinylidene fluoride membranes (Millipore, Billerica, Mass., USA). The membranes were blocked for $1 \mathrm{~h}$ at room temperature or overnight at $4{ }^{\circ} \mathrm{C}$ with $5 \%$ nonfat milk (Sangon Biotech, Shanghai, China). They were then incubated for $2 \mathrm{~h}$ at room temperature or overnight at $4^{\circ} \mathrm{C}$ with the following primary antibodies: rabbit anti-rabbit CD147 (1:400; Bioss, Beijing, China), cavia anti-rabbit IL-6 (1:400; Uscn Life science Inc., Wuhan, China), mouse anti-rabbit IL-10 (1: 500; Global Biotech, Shanghai, China), rabbit anti-rabbit TGF- $\beta 1$ -
1:500; Global Biotech), mouse anti-rabbit $\beta$-Actin (1:500; Boster). After 3 washes, membranes were incubated with horseradish peroxidase-conjugated secondary antibodies as follows: goat anti-rabbit (1:1,000, Boster), goat anti-guinea pig (1:1,000, Global Biotech), goat anti-mouse (1:1,000, Boster) for $1 \mathrm{~h}$ at room temperature.Proteinbandswerevisualized byenhanced chemiluminescence with an ECL Plus chemiluminescence detection kit (Sangon Biotech). The optical density of the protein bands was quantified by Gel Analysis v2.02 software (Clinx Science Instruments).

\section{Statistical Analysis}

All data were analyzed by SPSS 17.0 statistical software and presented as the mean \pm standard deviation (SD). Fisher's exact test was used to examine differences in mortality among the 4 groups and sizes of the atherosclerotic plaques. Analysis of variance (ANOVA) was used to determine differences in body weights between the groups. Differences among multiple groups were assessed with one-way ANOVA followed by the least significant difference test for serum lipids and expression of IL-6, IL-10, CD147, and TGF- $\beta$. Values of $\mathrm{p}<0.05$ were considered statistically significant.

\section{Results}

Transplantation of BMMNCs Did Not Affect Rabbit Mortality

At the end of each protocol, the mortalities of the 4 groups were $0(0 / 6), 0(0 / 6), 1(1 / 24)$ and $0(0 / 24)$, respectively. No significant differences were observed among the groups $(\mathrm{p}>0.05)$. The single rabbit died within the first $24 \mathrm{~h}$ after air desiccation.

\section{BMMNCs Treatment Did Not Affect the Level of Serum Lipid and Body Weight}

Exposure to a high-cholesterol diet for 8 weeks resulted in a significant increase in the body weights of rabbits in the sham operated, vehicle and BMMNC-treated groups compared to those in the naive group (naive: 2.01 \pm 0.27 ; sham: $2.78 \pm 0.54$; vehicle: $2.81 \pm 0.38$; BMMNC: $2.86 \pm 0.41 ; \mathrm{p}<0.05)$. However, no differences were observed between the 3 groups fed a high-cholesterol diet $(p>0.05)$. Two weeks after vehicle or BMMNC treatment, the rabbits that received a high-cholesterol diet had a significantly increased body weight compared to those in the naive group (naive: $2.33 \pm 0.31$; sham: $3.68 \pm 0.54$; vehicle: $3.85 \pm 0.66$; BMMNC: $3.94 \pm 0.52$; $\mathrm{p}>0.05$ ). BMMNC treatment did not increase the body weight of rabbits compared to vehicle treatment.

The serum levels of TC, TG, HDL and LDL in the sham operated and vehicle animals were significantly elevated compared with those in the naive group at 8,9 , or 10 weeks after a high-cholesterol diet. BMMNC treatment 


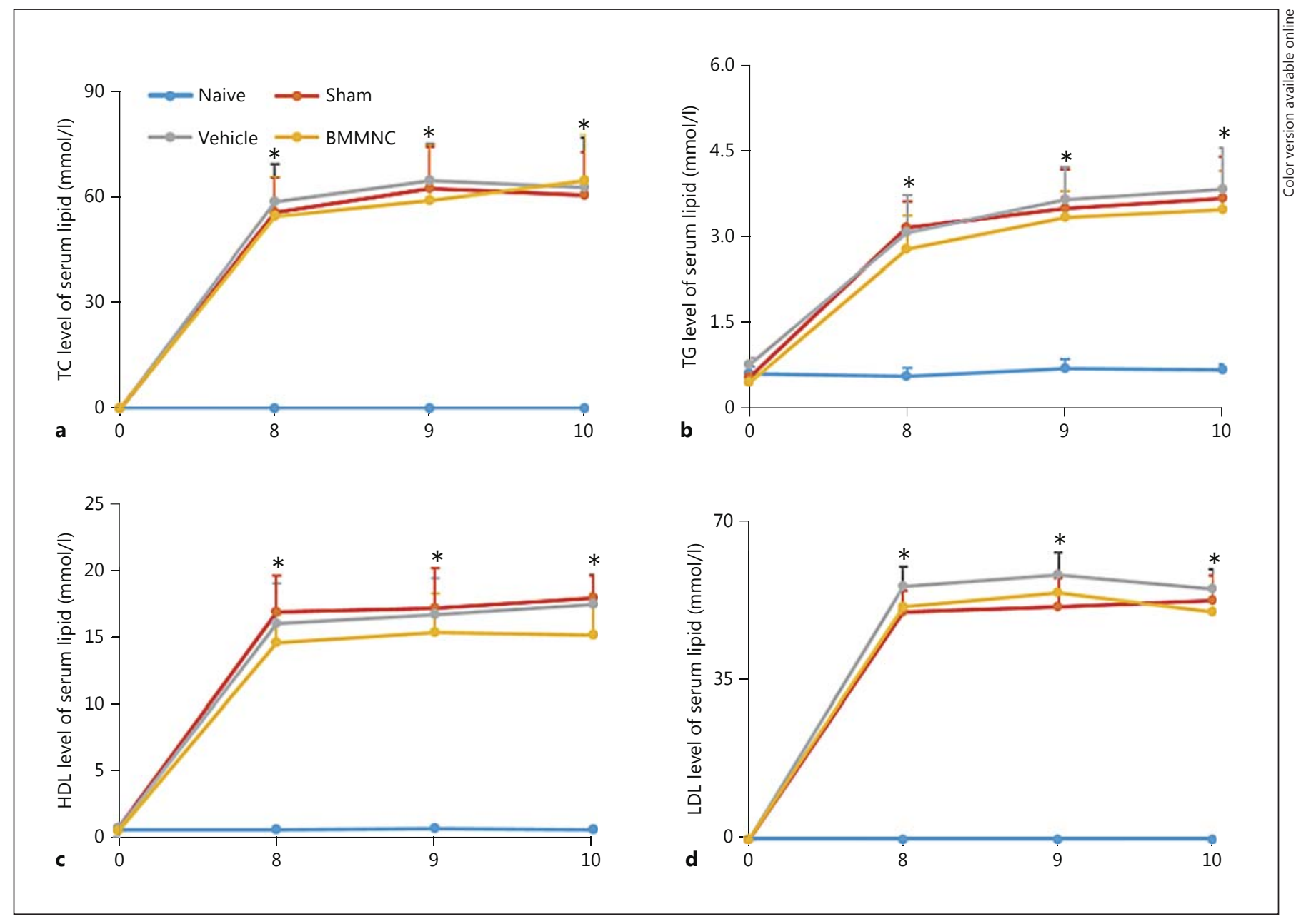

Fig. 1. BMMNC treatment did not affect serum lipid levels. A high-cholesterol diet for 10 weeks significantly increased levels of serum lipid TC (a), TG (b), HDL (c), and LDL (d) compared to the naive group. However, BMMNCs did not reduce these levels compared to the vehicle group 2 weeks after cell injection. ${ }^{*} \mathrm{p}<0.05$ versus naive group, $\mathrm{n}=6$ per group.

did not reduce the levels of TC, TG, HDL, and LDL compared to vehicle treatment at these observed time points (fig. 1a-d; $\mathrm{p}>0.05$ ).

\section{BMMNCs Reduced the Carotid Atherosclerotic Plaque Area}

Using an ultrasonographic imaging method, we found that the walls of carotid arteries in the naive and sham groups were smooth and no clear ultrasonographic plaque was found in these rabbits after 10 weeks of a highcholesterol diet (fig. 2a, b). In contrast, the carotid artery walls of rabbits in the vehicle and BMMNC groups were rough with visible hyperechoic plaques (fig. $2 c, d$ ). In addition, the average plaque diameter in the vehicle-treated group was significantly more than that in the BMMNC treatment group (fig. $2 \mathrm{c}-\mathrm{i}$ ). Representative vessels taken from comparable regions of the carotid from naive-, sham-, vehicle- and BMMNC-treated rabbits were stained with $\mathrm{HE}$ (fig. 2e-h). We found that autologous BMMNC transplantation significantly reduced areas of atherosclerotic plaque compared to the vehicle treatment group $(\mathrm{n}=6$ per group, $\mathrm{p}<0.05$; fig. $2 \mathrm{j}$ ). Moreover, BrdU-positive cells were found in the plaques of carotid arteries of BMMNC-treated rabbits on days 1 and 14 after the injection of cells (fig. 3b, c). The number of BrdU-positive cells were $\left(19.1 \pm 3.5 / \mathrm{mm}^{2}\right)$ and persisted at high levels for at least 14 days $\left(30.1 \pm 4.1 / \mathrm{mm}^{2} ; \mathrm{n}=6\right.$ per time point, day 14 vs. day $1, \mathrm{p}<0.05$; fig. $3 \mathrm{~d})$. 

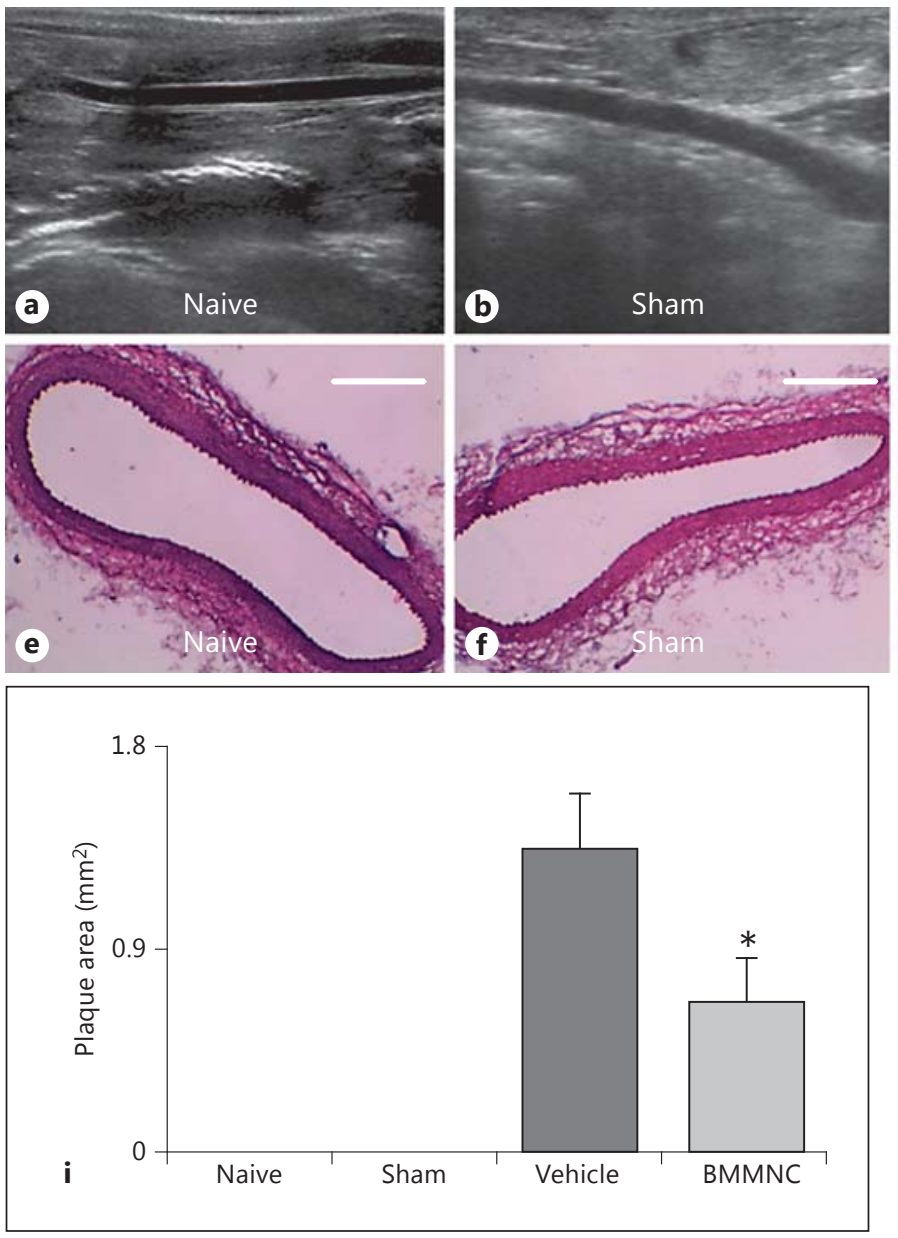

Fig. 2. BMMNC treatment reduced the carotid atherosclerotic plaque area. a-d Representative ultrasonographic images of left carotid arteries 2 weeks after BMMNC treatment. e-h Representative histological images of carotid sections stained with HE 2 weeks after BMMNC treatment. Scale bar $=200 \mu \mathrm{m}$. i, j Quantification
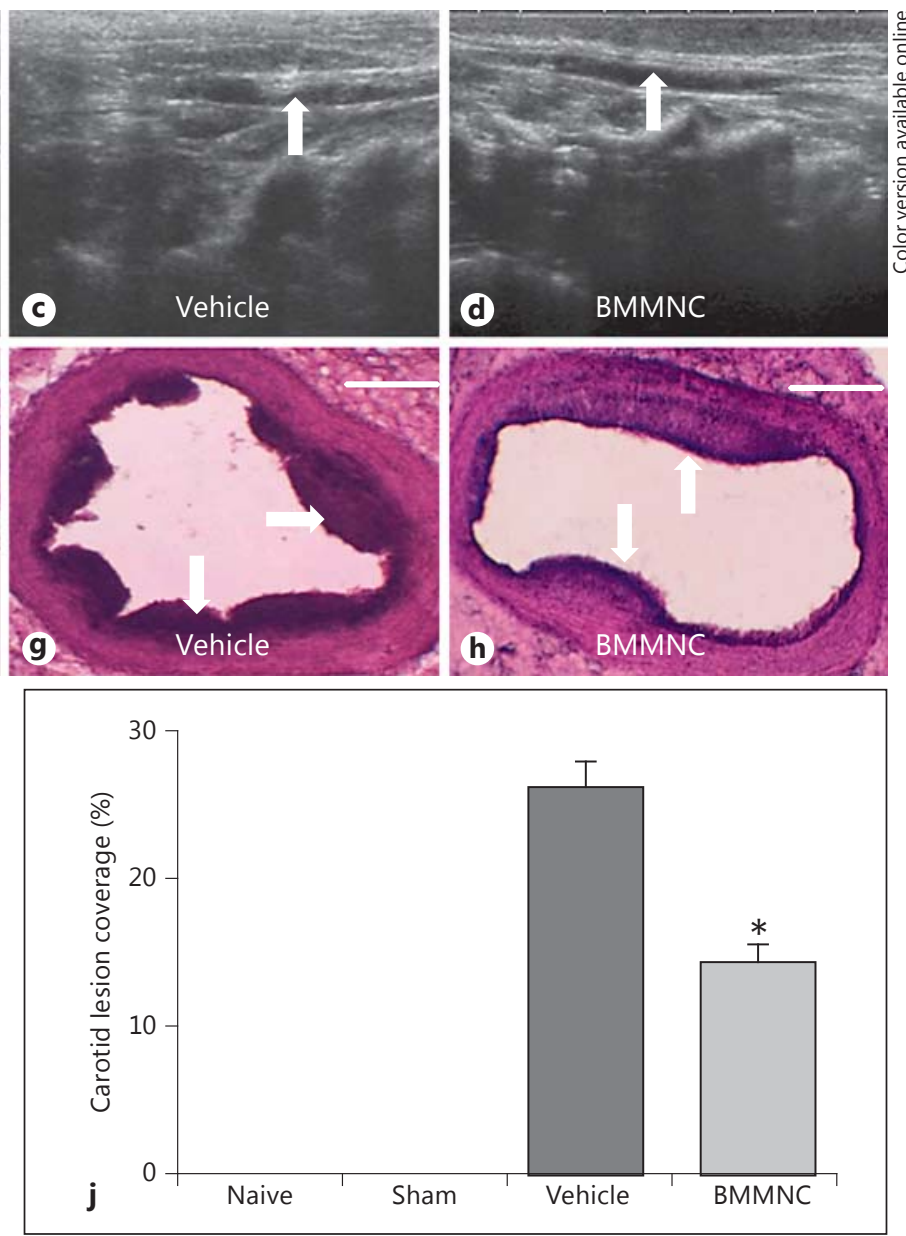

analysis of HE staining images showed that BMMNC treatment reduced the area of carotid atherosclerotic plaques. The arrows indicate the atherosclerotic plaque in the carotid artery wall. ${ }^{*} \mathrm{p}<$ 0.05 versus the vehicle-treated group, $\mathrm{n}=6$ per group.

group were significantly reduced on day 3 compared to that in the vehicle treatment group, and reached the lowest on day 7 and persisted at low values for at least 14 days after BMMNC treatment $(\mathrm{p}<0.05)$. Conversely, the levels of IL- 10 and TGF- $\beta$ in rabbits of the BMMNC transplantation group were significantly increased compared to those in the vehicle treatment group on day 1 and persisted at high values for at least 14 days after BMMNC treatment $(\mathrm{n}=6$ per time point, $\mathrm{p}<0.05$ vs. the corresponding vehicle group; fig. $4 \mathrm{a}-\mathrm{e}$ ). Moreover, Western blot analysis showed similar expression patterns of IL-6, IL-10, TGF- $\beta$, and CD147 to those found in RT-PCR ( $\mathrm{n}=6$ per time point, $\mathrm{p}<0.05$ vs. the corresponding vehicle group; fig. $4 \mathrm{f}, \mathrm{g}$ ). 

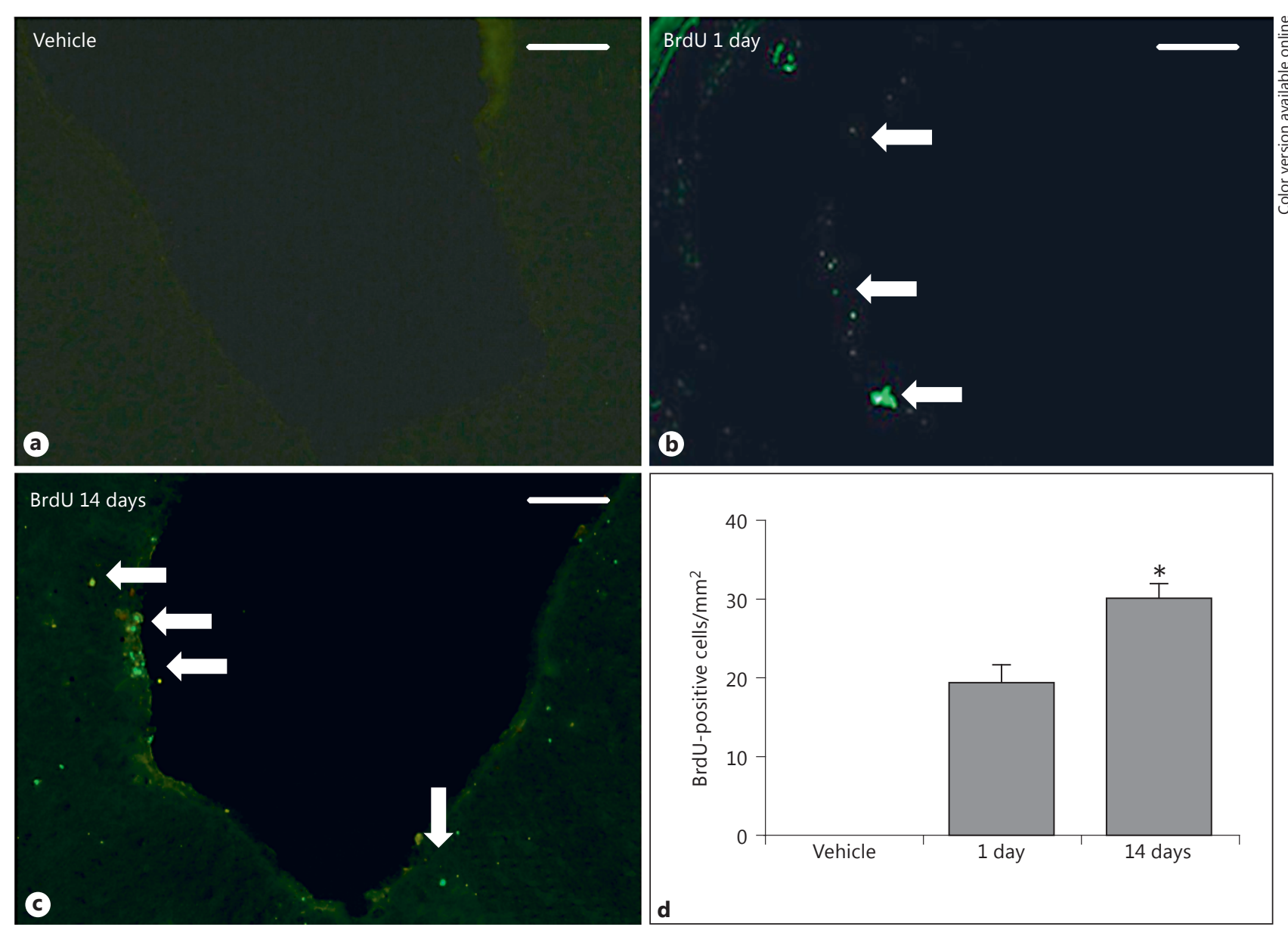

Fig. 3. Transplanted BMMNCs incorporated into carotid arteries. a-c Representative images of the vehicle- and BMMNC-treated groups on days 1 and 14 stained with anti-BrdU antibody (green; colors refer to the online version only). Scale bar $=100 \mu \mathrm{m}$. d Quantification showed that the number of BrdU-positive cells in atherosclerotic plaques on day 14 was more than that on day 1 after BMMNCs treatment. ${ }^{*} \mathrm{p}<0.05$ versus the vehicle-treated group on day $1, \mathrm{n}=6$ per time point. The arrows indicated BrdUpositive BMMNCs.

\section{Discussion}

Our research confirmed that transplanted autologous BMMNCs can migrate into atherosclerotic plaques in carotid arteries. Administered BMMNCs reduced the area of atherosclerotic plaques. The underlying mechanism involves the upregulation of anti-inflammatory cytokines (IL-10 and TGF- $\beta$ ) and the downregulation of proinflammatory mediators (IL-6 and CD147). Autologous $\mathrm{BMMNC}$ transplantation may represent a new therapeutic approach for treating AS.

Stromal cell-derived factor 1 (SDF-1) is expressed by VSMCs, endothelial cells and macrophages in atherosclerotic arteries [21]. Bone marrow contains a highly mobile population of CXCR4+ cells [22]. Through the CXCR4-
SDF-1 signaling pathway, BMMNCs can migrate into atherosclerotic lesions and have many vital effects on the progression of AS.

IL- 6 has been identified in the atherosclerotic lesion and its expression level has a significantly positive relationship with the extent and size of the plaques $[23,24]$. Overexpression of IL-6 may trigger a variety of immune responses and enhance fatty lesion development, leading to the aggravation of AS $[25,26]$. Furthermore, IL-6 is independently associated with the extent of AS in patients and can predict the future of coronary artery disease [27, 28]. CD147, expressed on many cell types, is the central inducer of MMP-9 derived from monocytes, which plays a key role in plaque rupture, resulting in myocardial infarction or stroke [7]. In our study, the content of CD147 


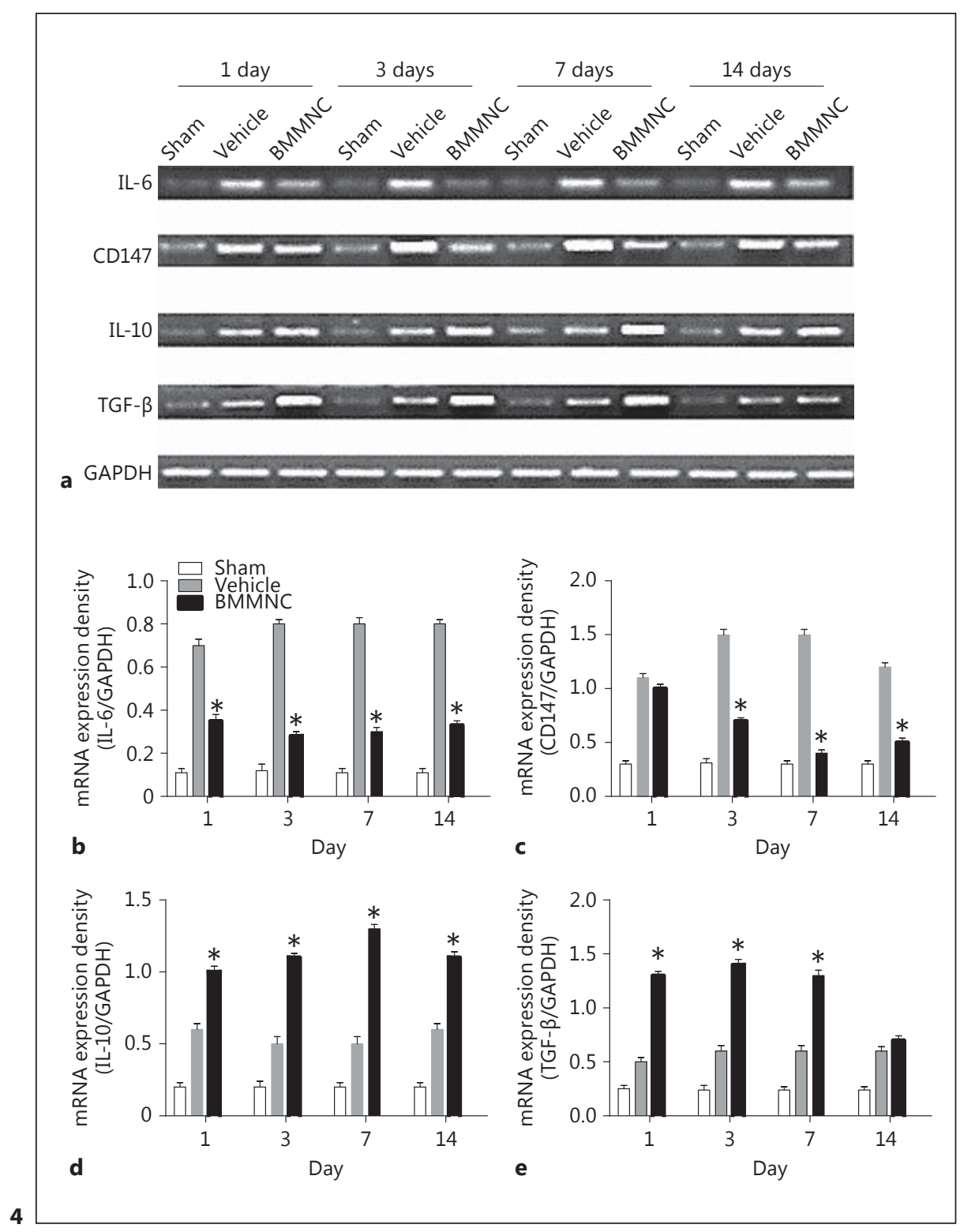

(For rest of figure and legend see next page.)

and IL-6 was significantly decreased by autologous BMMNC treatment. This result indicates that autologous BMMNC transplantation can suppress AS development by a reduction of proinflammatory mediators.

IL-10 is a pleiotropic cytokine which has a protective role in both atherogenesis and plaque instability [8]. It can be secreted by monocyte and Th2 subtype lymphocytes. Its prototypic role is to inhibit the activation of macrophages and subsequent secretion of MMPs and other inflammatory mediators, such as tumor necrosis factor- $\alpha$, monocyte chemotactic protein- 1 and intracellular adhesion molecule-1 [8]. Moreover, it can alter the lipid metabolism in macrophages and prevent the formation of foam cells [8]. TGF- $\beta$ plays a major role in the regulation of inflammation and immune responses by regulating macrophages or T cells [9]. Upregulation of TGF- $\beta$ not only reduces the formation of atherosclerotic plaque, but also induces a transition from a macrophagerich unstable plaque phenotype to a more stable, fibrous lesion $[9,29]$. In addition, TGF- $\beta$ plays an important role in maintaining the normal structure of the vessel wall via inhibiting VSMC proliferation, migration, and the differentiation of VSMCs [10]. In our experiment, the IL-10 and TGF- $\beta$ content in rabbits of the autologous BMMNC 
Fig. 4. BMMNC treatment downregulated the proinflammatory mediators IL- 6 and CD147 but increased the production of the anti-inflammatory cytokines IL-10 and TGF- $\beta$. RT-PCR (a) and Western blot (f) analysis of IL- 6 , IL-10, eNOS, and TGF- $\beta$ on days $1,3,7$, and 14 after BMMNC injection. b-e Quantification analysis of RTPCR bands showed BMMNC treatment reduced the expression of IL-6 and CD147 but increased the production of IL-10 and TGF- $\beta$. g-j Western blot results were consistent with the RT-PCR assay. ${ }^{*} \mathrm{p}<0.05$ versus corresponding vehicle groups, $\mathrm{n}=6$ per group.

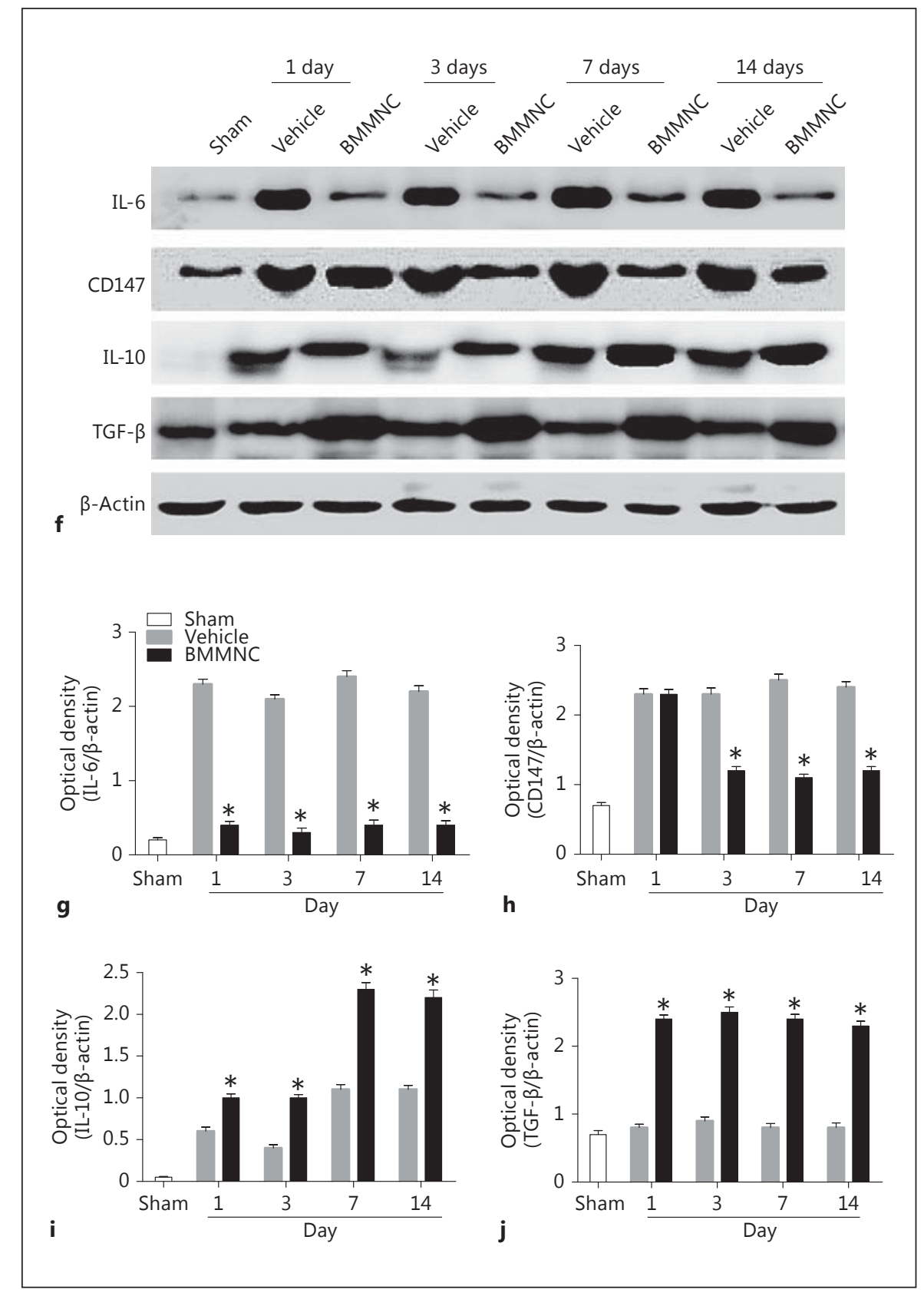

transplantation group was significantly elevated compared to those in the vehicle-treated group. These results suggest that autologous BMMNC transplantation can inhibit the development of AS by upregulation of the expression of IL-10 and TGF- $\beta$. BMMNCs consist of various subpopulations of cells, including regulatory $\mathrm{T}$ (Treg) cells and type 2 macrophages (M2) [30]. These cells possess immune regulatory capacity by secreted IL-10 and TGF- $\beta$. In addition, AS reduces the number of peripheral Treg cells, which weakens the resistance to inflammatory

Autologous BMMNC Treatment for Atherosclerosis responses. Treg cells and M2 cells derived from BMMNCs can make up for the loss. The increased anti-inflammatory mediators may be derived from the secretion of autologous BMMNCs.

Most previous studies used allogeneic or syngeneic cells to explore the effects of BMMNCs on AS $[16,18,31]$. Indeed, transplantation of autologous BMMNCs may have different effects on recipients compared to allogeneic or syngeneic treatment $[32,33]$. Firstly, allogeneic or syngeneic BMMNCs are not harvested from the recipient 
and immunologic rejections may be present to some degree $[32,33]$, and may produce inflammatory cytokines and accelerate the process of AS. In contrast, immunologic rejections do not occur in the process of transplantation of autologous cells [34]. Secondly, autologous BMMNCs are obtained after AS, the compositions of BMMNCs may change and tend to have anti-inflammatory properties, similar to the effects of stroke on bone marrow $[35,36]$. Together, our data support the belief that the transplantation of autologous BMMNCs may have protective effects against AS.

\section{Acknowledgements}

This work was supported by the National Natural Science Foundation of China (No. 81371583) and NIH (R01AT007317, R01NS078026).

\section{Disclosure Statement}

All of the authors have no conflicts of interest to report.

\section{References}

1 Ylä-Herttuala S, Bentzon JF, Daemen M, Falk E, Garcia-Garcia HM, Herrmann J, Hoefer I, Jukema JW, Krams R, Kwak BR: Stabilisation of atherosclerotic plaques. Thromb Haemost 2011;106:1-19.

2 Khakoo AY, Finkel T: Endothelial progenitor cells. Annu Rev Med 2005;56:79-101.

3 Schmidt-Lucke C, Rössig L, Fichtlscherer S, Vasa M, Britten M, Kämper U, Dimmeler S, Zeiher AM: Reduced number of circulating endothelial progenitor cells predicts future cardiovascular events proof of concept for the clinical importance of endogenous vascular repair. Circulation 2005;111:2981-2987.

4 Chaabane C, Coen M, Bochaton-Piallat ML: Smooth muscle cell phenotypic switch: implications for foam cell formation. Curr Opin Lipidol 2014;25:374-379.

5 Tedgui A, Mallat Z: Cytokines in atherosclerosis: pathogenic and regulatory pathways. Physiol Rev 2006;86:515-581.

6 Nathan C: Points of control in inflammation. Nature 2002;420:846-852.

7 Iacono KT, Brown AL, Greene MI, Saouaf SJ: CD147 immunoglobulin superfamily receptor function and role in pathology. Exp Mol Pathol 2007;83:283-295.

8 Han X, Boisvert WA: Interleukin-10 protects against atherosclerosis by modulating multiple atherogenic macrophage function. Thromb Haemost 2015;113:505-512.

9 Li MO, Flavell RA: TGF- $\beta$ : a master of all T cell trades. Cell 2008;134:392-404.

10 Dhaouadi N, Li JY, Feugier P, Gustin MP, Dab H, Kacem K, Bricca G, Cerutti C: Computational identification of potential transcriptional regulators of TGF- $\beta 1$ in human atherosclerotic arteries. Genomics 2014;103:357370.

11 Wang Z-X, Li D, Cao J-X, Liu Y-S, Wang M, Zhang X-Y, Li J-L, Wang H-B, Liu J-L, Xu B-L: Efficacy of autologous bone marrow mononuclear cell therapy in patients with peripheral arterial disease. J Atheroscler Thromb 2014;21:1183-1196.
12 Delewi R, van der Laan AM, Robbers LF, Hirsch A, Nijveldt R, van der Vleuten PA, Tijssen JG, Tio RA, Waltenberger J, ten Berg JM: Long term outcome after mononuclear bone marrow or peripheral blood cells infusion after myocardial infarction. Heart 2015;101: 363-368.

13 Taguchi A, Sakai C, Soma T, Kasahara Y, Stern DM, Kajimoto K, Ihara M, Daimon T, Yamahara K, Doi K: Intravenous autologous bone marrow mononuclear cell transplantation for stroke: phase1/2a clinical trial in a homogeneous group of stroke patients. Stem Cells Dev 2015;24:2207-2218.

14 Wang J, Yu L, Jiang C, Chen M, Ou C, Wang $\mathrm{J}$ : Bone marrow mononuclear cells exert longterm neuroprotection in a rat model of ischemic stroke by promoting arteriogenesis and angiogenesis. Brain Behav Immun 2013;34: 56-66.

15 Simari RD, Pepine CJ, Traverse JH, Henry TD, Bolli R, Spoon DB, Yeh E, Hare JM, Schulman IH, Anderson RD, Lambert C, Sayre SL, Taylor DA, Ebert RF, Moye LA: Bone marrow mononuclear cell therapy for acute myocardial infarction: a perspective from the cardiovascular cell therapy research network. Circ Res 2014;114:1564-1568.

16 Arnold R, Villa A, Gutiérrez H, Sánchez PL Gimeno F, Fernández ME, et al: Absence of accelerated atherosclerotic disease progression after intracoronary infusion of bone marrow derived mononuclear cells in patients with acute myocardial infarction - angiographic and intravascular ultrasound - results from the Terapia Celular Aplicada al Miocardio pilot study. Am Heart J 2010;159:1154. e1-8.

17 Fledderus JO, van Oostrom O, de Kleijn DP, den Ouden K, Penders AF, Gremmels H, de Bree P, Verhaar MC: Increased amount of bone marrow-derived smooth muscle-like cells and accelerated atherosclerosis in diabetic apoe-deficient mice. Atherosclerosis 2013; 226:341-347.
18 Mees B, Recalde A, Loinard C, Tempel D, Godinho M, Vilar J, van Haperen R, Levy B, de Crom R, Silvestre JS: Endothelial nitric oxide synthase overexpression restores the efficiency of bone marrow mononuclear cell-based therapy. Am J Pathol 2011;178:55-60.

19 Chen X, Ren S, Ma MG, Dharmalingam S, Lu L, Xue M, Ducas J, Shen GX: Hirulog-like peptide reduces restenosis and expression of tissue factor and transforming growth factor- $\beta$ in carotid artery of atherosclerotic rabbits. Atherosclerosis 2003;169:31-40.

20 Yamashita A, Zhao Y, Matsuura Y, Yamasaki K, Moriguchi-Goto S, Sugita C, Iwakiri T, Okuyama N, Koshimoto C, Kawai K: Increased metabolite levels of glycolysis and pentose phosphate pathway in rabbit atherosclerotic arteries and hypoxic macrophage. PLoS One 2014;9:e86426.

21 Gear AR, Camerini D: Platelet chemokines and chemokine receptors: linking hemostasis, inflammation, and host defense. Microcirculation 2003;10:335-350.

22 Kucia M, Ratajczak J, Ratajczak MZ: Bone marrow as a source of circulating $\mathrm{CXCR} 4^{+}$ tissue-committed stem cells. Biol Cell 2005; 97:133-146.

23 Sukovich DA, Kauser K, Shirley FD, DelVecchio V, Halks-Miller M, Rubanyi GM: Expression of interleukin-6 in atherosclerotic lesions of male ApoE-knockout mice: inhibition by $17 \beta$-estradiol. Arterioscler Thromb Vasc Biol 1998;18:1498-1505.

24 Seino Y, Ikeda U, Ikeda M, Yamamoto K, Misawa Y, Hasegawa T, Kano S, Shimada K: Interleukin 6 gene transcripts are expressed in human atherosclerotic lesions. Cytokine 1994;6:87-91.

25 Huber SA, Sakkinen P, Conze D, Hardin N, Tracy R: Interleukin-6 exacerbates early atherosclerosis in mice. Arterioscler Thromb Vasc Biol 1999;19:2364-2367. 
26 Lin G, Shi X, Chen S, Lei L, You X, Huang M, Luo L, Li Y, Zhao X, Yan F: Effects of microamounts of Porphyromonas gingivalis lipopolysaccharide on rabbit inflammatory immune response and development of atherosclerosis. J Periodontal Res 2015;50:356-362.

27 Kablak-Ziembicka A, Przewlocki T, Stepien E, Pieniazek P, Rzeznik D, Sliwiak D, Komar M, Tracz W, Podolec P: Relationship between carotid intima-media thickness, cytokines, atherosclerosis extent and a two-year cardiovascular risk in patients with arteriosclerosis. Kardiol Pol 2011;69:1024-1031.

28 Harris TB, Ferrucci L, Tracy RP, Corti MC, Wacholder S, Ettinger WH, Heimovitz H, Cohen HJ, Wallace R: Associations of elevated interleukin-6 and C-reactive protein levels with mortality in the elderly. Am J Med 1999; 106:506-512.

29 Grainger DJ: Transforming growth factor $\beta$ and atherosclerosis: so far, so good for the protective cytokine hypothesis. Arterioscler Thromb Vasc Biol 2004;24:399-404.
30 Zaiss MM, Axmann R, Zwerina J, Polzer K, Gückel E, Skapenko A, Schulze-Koops H, Horwood N, Cope A, Schett G: Treg cells suppress osteoclast formation: a new link between the immune system and bone. Arthritis Rheum 2007;56:4104-4112.

31 Silvestre J-S, Gojova A, Brun V, Potteaux S, Esposito B, Duriez M, Clergue M, Le RicousseRoussanne S, Barateau V, Merval R: Transplantation of bone marrow-derived mononuclear cells in ischemic apolipoprotein Eknockout mice accelerates atherosclerosis without altering plaque composition. Circulation 2003; 108:2839-2842.

32 Wang D, Yu Y, Haarberg K, Fu J, Kaosaard K, Nagaraj S, Anasetti C, Gabrilovich D, Yu X-Z: Dynamic change and impact of myeloid-derived suppressor cells in allogeneic bone marrow transplantation in mice. Biol Blood Marrow Transplant 2013;19:692-702.
33 Shainer R, Azar Y, Almogi-Hazan O, Bringer R, Compton SR, Paidas MJ, Barnea ER, Or R: Immune regulation and oxidative stress reduction by preimplantation factor following syngeneic or allogeneic bone marrow transplantation. Conf Papers Sci 2013;2013: 718031.

34 Njerve IU, Solheim S, Lunde K, Hoffmann P, Arnesen H, Seljeflot I: Fractalkine levels are elevated early after PCI-treated ST-elevation myocardial infarction; no influence of autologous bone marrow derived stem cell injection. Cytokine 2014;69:131-135.

35 Wang J, Yu L, Jiang C, Fu X, Liu X, Wang M, Ou C, Cui X, Zhou C, Wang J: Cerebral ischemia increases bone marrow $\mathrm{CD} 4^{+} \mathrm{CD} 25^{+}$ $\mathrm{FoxP}^{+}$regulatory $\mathrm{T}$ cells in mice via signals from sympathetic nervous system. Brain Behav Immun 2015;43:172-183.

36 Courties G, Herisson F, Sager HB, Heidt T, Ye Y, Wei Y, Sun Y, Severe N, Dutta P, Scharff J: Ischemic stroke activates hematopoietic bone marrow stem cells. Circ Res 2015;116:407417. 